\title{
CONCENTRATION OF CYLINDRICAL WIGNER MEASURES
}

\author{
MARCO FALCONI
}

\begin{abstract}
In this note we aim to characterize the cylindrical Wigner measures associated to regular quantum states in the Weyl $\mathrm{C}^{*}$-algebra of canonical commutation relations. In particular, we provide conditions at the quantum level sufficient to prove the concentration of all the corresponding cylindrical Wigner measures as Radon measures on suitable topological vector spaces. The analysis is motivated by variational and dynamical problems in the semiclassical study of bosonic quantum field theories.
\end{abstract}

\section{Introduction}

In this brief note, we discuss the concentration of cylindrical Wigner measures. Cylindrical Wigner measures on a topological vector space $V$ have been introduced in [12] as the classical limit points, in a suitable topology, of regular quantum states in the Weyl $\mathrm{C}^{*}$-algebra of canonical commutation relations $\mathbf{W}_{h}\left(V^{\prime}, \sigma\right)$ - where $V^{\prime}$ is the continuous dual of some topological vector space $V$. The dual $V^{\prime}$ is endowed with a symplectic form, and it is interpreted as the classical phase space. Any cylindrical measure on a (infinite dimensional) topological vector space $V$ is a Borel Radon measure on a "bigger" space $\bar{V}$ (see Section 3, or [21], for additional details). However, the space $\bar{V}$ is usually too large, in the sense that it is not manageable to study dynamical or variational problems in the semiclassical or mean field analysis of bosonic many-particle systems and fields. In applications [see e.g. 1, 2, 4-7, 11, 18-20, and references thereof contained] the attention is often restricted, due to the physical properties of the system, to quantum states whose corresponding Wigner measures are concentrated as Radon measures on some manageable (e.g. separable Hilbert) topological vector space. It is therefore natural to ask for conditions on regular quantum states that are sufficient to yield concentration, on some given space, of all the corresponding Wigner measures.

Date: July 23, 2018.

2010 Mathematics Subject Classification. Primary: 81Q20, 81S05. Secondary: 46L99, 47L90.

Key words and phrases. Semiclassical analysis, Cylindrical Wigner measures, Quantum Field Theory.

The author acknowledges the support of MIUR through the FIR grant 2013 Condensed Matter in Mathematical Physics (Cond-Math) (code RBFR13WAET). The final version of this preprint is to appear in Communications in Contemporary Mathematics (C) 2017 World Scientific Publishing Company. 
In Section 3.1, we provide sufficient conditions to prove concentration in separable complex Hilbert spaces (with inner product compatible with the symplectic form $\sigma$ ); in Section 3.2 we provide sufficient conditions to prove concentration on continuous duals of nuclear spaces (with any locally convex topology between the ultraweak and the Mackey topology); in Section 3.3 we provide sufficient conditions to prove concentration in duals of Banach spaces, endowed with the ultraweak topology. For the Weyl $\mathrm{C}^{*}$-algebra $\mathbf{W}_{h}\left(\mathbb{F} \mathscr{H}, \operatorname{Im}\langle\cdot, \cdot\rangle_{\mathscr{H}}\right)$ - built on the classical separable Hilbert phase space $\mathbb{F} \mathscr{H}$ associated to a complex Hilbert space $\mathscr{H}$ - sufficient conditions on Fock-normal quantum states to prove concentration on $\mathscr{H}$ (and on suitable Hilbert spaces included in $\mathscr{H}$ ) have been given in [3, 5]. Theorem 3.3 can be seen as a generalization of the aforementioned results, to more general regular quantum states on arbitrary Weyl $C^{*}$-algebras. Such generalization is motivated by concrete problems in the variational and dynamical semiclassical analysis of bosonic quantum field theories, as explained in Sections 1.1 and 3.4. Our analysis is based on the "cylindrical" (finite-dimensional) pseudodifferential calculus for bosonic quantum field theories, that is outlined in Section 2.

Remark. Theorems 3.3, 3.4, and 3.8 can be easily adapted to regular quantum states in the tensor product of the $\mathrm{C}^{*}$-algebra of canonical commutation relations $\mathbf{W}_{h}\left(V^{\prime}, \sigma\right)$ with another $\mathrm{C}^{*}$-algebra $\mathfrak{A}$ describing additional degrees of freedom of the physical system [see 12]. The only difference is that the Wigner measures are, in this case, vector-valued and take values in the space $\mathfrak{A}_{+}^{\prime}$ of (positive) states acting on $\mathfrak{A}$.

1.1. Motivation. This technical note is motivated by some problems we encountered in the study of the semiclassical properties of bosonic quantum systems $[1,11]$. Let us discuss briefly a concrete case. Let $d \geq 2$, and consider a selfadjoint and bounded from below operator $H_{h}$ on the symmetric Fock space

$$
\Gamma_{\mathrm{s}}\left(L^{2}\left(\mathbb{R}^{d}\right)\right)=\bigoplus_{n \in \mathbb{N}} L_{n}^{2}=\mathbb{C} \bigoplus_{n \in \mathbb{N}_{*}} \underbrace{L^{2} \otimes_{\mathrm{s}} \cdots \otimes_{\mathrm{s}} L^{2}}_{n} .
$$

$H_{h}$ is the quantization of some classical symbol $\mathscr{E}$, and it is defined on the domain $D\left(\mathrm{~d} \Gamma_{h}(|k|)\right)$, where

$$
L_{n}^{2} \ni\left(\mathrm{d} \Gamma_{h}(|k|) \psi_{h}\right)_{n}\left(k_{1}, \ldots, k_{n}\right)=h \sum_{j=1}^{n}\left|k_{j}\right| \psi_{h, n}\left(k_{1}, \ldots, k_{n}\right) .
$$

Throughout the paper, $h \in(0,1)$ denotes the semiclassical parameter. The second quantized operator $\mathrm{d} \Gamma_{h}(|k|)$ is the free energy of massless bosonic scalar fields, e. $g$. radiation. Suppose now that we want to investigate the convergence, as $h \rightarrow 0$, of the ground state energy $\underline{\sigma}\left(H_{h}\right)$. Let $j \in \mathbb{N}$, and $\psi_{h}^{(j)} \in D\left(\mathrm{~d} \Gamma_{h}(|k|)\right)$ be a vector 
in a normalized minimizing sequence of $H_{h}$. Since $\left(\psi_{h}^{(j)}\right)_{h \in(0,1)}$ is norm-bounded uniformly with respect to $h$, its cluster points are cylindrical Wigner measures [see 12]. If all the cluster points concentrate as measures in the classical energy space $\mathscr{F} \dot{H}^{1 / 2}\left(\mathbb{R}^{d}\right)=L^{2}\left(\mathbb{R}^{d},|k| \mathrm{d} k\right)$, we can use the information to prove the convergence

$$
\lim _{h \rightarrow 0} \underline{\sigma}\left(H_{h}\right)=\inf _{\alpha \in \mathscr{F} \dot{H}^{1 / 2}} \mathscr{E}(\alpha) ;
$$

see [1] for a more detailed explanation of the strategy. It is well-known [3] that

$$
\left\langle\varphi_{h}, N_{h} \varphi_{h}\right\rangle_{\Gamma_{\mathrm{s}}}=\left\langle\varphi_{h}, \mathrm{~d} \Gamma_{h}(1) \varphi_{h}\right\rangle_{\Gamma_{\mathrm{s}}} \leq \mathcal{K}
$$

is sufficient to prove concentration of the cluster points of $\left(\psi_{h}\right)_{h \in(0,1)}$ in $L^{2}\left(\mathbb{R}^{d}\right)$. However, for $\left(\psi_{h}^{(j)}\right)_{h \in(0,1)}$ we have the sole information

$$
\left\langle\psi_{h}^{(j)}, \mathrm{d} \Gamma_{h}(|k|) \psi_{h}^{(j)}\right\rangle_{\Gamma_{\mathrm{s}}} \leq \mathcal{K} .
$$

In addition, since the symmetric difference $L^{2} \ominus \mathscr{F} \dot{H}^{1 / 2}$ is not empty, there are families of quantum states $\left(\varphi_{h}\right)_{h \in(0,1)}$ that converge to Wigner measures that are Radon on $\mathscr{F} \dot{H}^{1 / 2}$ and concentrated outside of $L^{2}$. Therefore it is not possible to restrict the analysis to vectors that satisfy both (1) and (2); and in fact the miminimizing sequence might not satisfy (1). As we will prove in Theorem 3.3, bound (2) is sufficient to have concentration of all the corresponding cylindrical Wigner measures in $\mathscr{F} \dot{H}^{1 / 2}$. Let us formulate here the result explicitly, and give an outline of the proof. This would serve to explain the key idea behind the proof of the more general Theorem 3.3.

Proposition. Let $\left(\varphi_{h}\right)_{h \in(0,1)} \subset \Gamma_{\mathrm{S}}\left(L^{2}\left(\mathbb{R}^{d}\right)\right)$. Suppose there exists $\mathcal{K}>0$ such that

$$
\left\langle\varphi_{h}, \mathrm{~d} \Gamma_{h}(|k|) \varphi_{h}\right\rangle_{\Gamma_{\mathrm{s}}} \leq \mathcal{K}
$$

Then any Wigner measure that is a cluster point of $\left(\varphi_{h}\right)_{h \in(0,1)}$ is a Borel Radon measure on $\mathscr{F} \dot{H}^{1 / 2}\left(\mathbb{R}^{d}\right)$.

Sketch of the proof. Let $G \subset L^{2}$ be a finite dimensional subspace with orthonormal basis $\left\{g_{j}\right\}_{j \in\{0, \ldots, n\}}$; there is a canonical identification of the Fock-space creation and annihilation operators $\left\{a_{h}^{\#}\left(g_{j}\right)\right\}_{j \in\{0, \ldots, n\}}$ with the standard creation and annihilation operators associated to the Heisenberg group [see e.g. 3]

$$
\mathbf{H}\left(\mathbb{F} G, \operatorname{Im}\langle\cdot, \cdot\rangle_{2}\right)=\mathbf{H}\left(\operatorname{span}_{\mathbb{R}}\left(g_{1}, i g_{1}, \ldots, g_{n}, i g_{n}\right), \operatorname{Im}\langle\cdot, \cdot\rangle_{2}\right) .
$$

Since we want to prove concentration of measures on a different space, namely $\mathscr{F} \dot{H}^{1 / 2}$, we need to make an alternative identification. This is the key step in the proof, hence let us explain in detail how to make the identification. 
Let $\left(e_{j}\right)_{j \in \mathbb{N}} \subset \mathscr{S}\left(\mathbb{R}^{d}\right)$ be an orthonormal basis of the Hilbert space $\mathscr{F} \dot{H}^{1 / 2}\left(\mathbb{R}^{d}\right)$. Then

$$
E_{R}=\operatorname{span}_{\mathbb{C}}\left\{e_{0}, e_{1}, \ldots, e_{R}\right\}, R \in \mathbb{N}
$$

is an increasing sequence of finite dimensional complex Hilbert spaces ordered by inclusion, such that $\bigcup_{R \in \mathbb{N}} E_{R}=\mathscr{F} \dot{H}^{1 / 2}$. Equivalently, each $E_{R}$ can be seen as a real symplectic space $\mathbb{F} E_{R}$ of dimension $2 R$ with symplectic form $\sigma_{E}(\cdot, \cdot)=$ $\operatorname{Im}\langle\cdot, \cdot\rangle_{\mathscr{F} \dot{H}^{1 / 2}}$. Now let $\left(\varepsilon_{j}\right)_{j \in \mathbb{N}} \subset \mathscr{S}\left(\mathbb{R}^{d}\right)$ be defined by $\varepsilon_{j}(k)=|k|^{1 / 2} e_{j}(k)$, and

$$
\mathcal{E}_{R}=\operatorname{span}_{\mathbb{C}}\left\{\varepsilon_{0}, \varepsilon_{1}, \ldots, \varepsilon_{R}\right\}, \sigma_{\mathcal{E}}(\cdot, \cdot)=\operatorname{Im}\langle\cdot, \cdot\rangle_{2} .
$$

Clearly, $\left(\mathbb{F} E_{R}, \sigma_{E}\right)$ and $\left(\mathbb{F} \mathcal{E}_{R}, \sigma_{\mathcal{E}}\right)$ are the same symplectic spaces under the identification $e_{j} \mapsto \varepsilon_{j}$ (or equivalently $E_{R}$ and $\mathcal{E}_{R}$ are the same complex Hilbert spaces).

Consider now the finite-dimensional Heisenberg group $\mathbf{H}\left(\mathbb{F} E_{R}, \sigma_{E}\right)$; since $E_{R} \subset$ $L^{2}$, the Heisenberg group has a unitary representation in $\Gamma_{\mathrm{S}}\left(L^{2}\right)$, given by

$$
e^{i t} W_{h}\left(|\cdot|^{1 / 2} e(\cdot)\right),(e, t) \in E_{R} \times \mathbb{R} ;
$$

where the Weyl operators are customarily defined by means of the usual creation and annihilation operators

$$
W_{h}(f)=e^{i\left(a_{h}^{*}(f)+a_{h}(f)\right)}, \quad \forall f, g \in L^{2}\left[a_{h}(f), a_{h}^{*}(g)\right]=h\langle f, g\rangle_{2} .
$$

On the other hand, $\mathrm{d} \Gamma_{h}(|k|)$ is the Wick quantization of $\|z\|_{\mathscr{F} \dot{H}^{1 / 2}}^{2}$. It follows that

$$
\mathrm{d} \Gamma_{h}(|k|)=\sum_{j \in \mathbb{N}} \mathbf{a}_{h}^{*}\left(\varepsilon_{j}\right) \mathbf{a}_{h}\left(\varepsilon_{j}\right)=\mathbf{N}_{h},
$$

where the modified creation and annihilation operators $\mathbf{a}_{h}^{\#}(\cdot)$ are defined by

$$
\begin{aligned}
\left(\mathbf{a}_{h}(f) \psi_{h}\right)_{n}\left(k_{1}, \ldots, k_{n}\right) & =\sqrt{h(n+1)} \int_{\mathbb{R}^{d}}|k|^{1 / 2} f(k) \psi_{h, n+1}\left(k, k_{1}, \ldots, k_{n}\right) \mathrm{d} k \\
\left(\mathbf{a}_{h}^{*}(f) \psi_{h}\right)_{n}\left(k_{1}, \ldots, k_{n}\right) & =\sqrt{h / n} \sum_{i=1}^{n}\left|k_{i}\right|^{1 / 2} f\left(k_{i}\right) \psi_{h, n-1}\left(k_{1}, \ldots, \underline{k}_{j}, \ldots, k_{n}\right)
\end{aligned}
$$

where $\underline{k}$ means that the variable is missing. The restriction of $\|z\|_{\mathscr{F}^{1 / 2}}^{2}$ to $E_{R}$ takes the form

$$
\left.\left.\sum_{j=0}^{R}\left|\int_{\mathbb{R}^{d}}\right| k\right|^{1 / 2} \bar{z}(k) \varepsilon_{j}(k) \mathrm{d} k\right|^{2},
$$

and therefore its Wick quantization is

$$
\mathrm{d} \Gamma_{h}(|k|)_{R}=\sum_{j=0}^{R} \mathbf{a}_{h}^{*}\left(\varepsilon_{j}\right) \mathbf{a}_{h}\left(\varepsilon_{j}\right)=\mathbf{N}_{h, R} .
$$


In addition, by means of the modified creation and annihilation operators it is possible to write the Weyl operators $W_{h}\left(|\cdot|{ }^{1 / 2} e(\cdot)\right), e \in E_{R}$, as

$$
W_{h}\left(|\cdot|^{1 / 2} e(\cdot)\right)=e^{i\left(\mathbf{a}_{h}^{*}(e)+\mathbf{a}_{h}(e)\right)} .
$$

Therefore $\left\{\mathbf{a}_{h}^{\#}\left(e_{j}\right)\right\}_{j \in\{0, \ldots, R\}}$ are identified with the usual creation and annihilation operators on the finite-dimensional Fock representation $\Gamma_{\mathrm{s}}\left(\mathbb{C}^{R+1}\right)$, and $\mathbb{C}^{R+1} \cong E_{R}$ as complex Hilbert spaces.

Let us remark again that the identifications above are the key ingredient that allows us to use the standard tools of pseudodifferential calculus associated to the Heisenberg group $\mathbf{H}\left(\mathbb{F} E_{R}, \sigma_{E}\right)$. The result is then proved combining such finite-dimensional pseudodifferential techniques with a Prokhorov-type tightness argument on the projective family of cylindrical measures, exploiting the fact that $\bigcup_{R \in \mathbb{N}} E_{R}=\mathscr{F} \dot{H}^{1 / 2}$. The details can be found in the proof of Theorem 3.3.

Other applications of Theorem 3.3 that are important for the semiclassical analysis of physical problems in bosonic quantum field theory will be given in Section 3.4.

\section{Cylindrical SEMiClassical ANAlysis}

Let $V$ be a real locally convex space. A cylindrical measure on $V$ is a projective family $M=\left(\mu_{\Phi}\right)_{\Phi \in F(V)}$ of finite Borel Radon measures on $V / \Phi$, where $F(V)$ is the set of closed subspaces of $V$ with finite codimension. To every $\Phi \in F(V)$, there corresponds the finite dimensional polar (orthogonal) $\Phi^{\circ} \subset V^{\prime}$, where $V^{\prime}$ is the continuous dual of $V$.

Definition 2.1 (Cylindrical symbol). A function $f: V \rightarrow \mathbb{C}$ is a cylindrical symbol with base $\Phi \in F(V)$ iff there exists a function $f_{\Phi}: V / \Phi \rightarrow \mathbb{C}$ such that

$$
\forall v \in V, f(v)=f_{\Phi}\left([v]_{\Phi}\right) .
$$

Let $f$ be a cylindrical symbol, $f_{\Phi}$ the corresponding finite dimensional function. If $f_{\Phi}$ is measurable, it is possible to define its cylindrical integral with respect to any cylindrical measure $\mu$ :

$$
\int_{V}^{(\mathrm{cyl})} f(v) \mathrm{d} M(v)=\int_{V / \Phi} f_{\Phi}(w) \mathrm{d} \mu_{\Phi}(w) .
$$

If we denote by $p_{\Phi}: V \rightarrow V / \Phi$ the canonical projection onto the equivalence classes, ${ }^{\mathrm{t}} p_{\Phi}:(V / \Phi)^{\prime} \rightarrow \Phi^{\circ}$ is an isomorphism. It follows that for any $f_{\Phi} \in$ $L^{1}(V / \Phi)$,

$$
\int_{V}^{(\text {cyl })} f(v) \mathrm{d} M(v)=\int_{V / \Phi} f_{\Phi}(w) \mathrm{d} \mu_{\Phi}(w)=\int_{\Phi^{\circ}} \hat{f}_{\Phi}(\xi) \hat{M}(2 \pi \xi) \mathrm{d} L_{\Phi^{\circ}}(\xi) .
$$


Here $\hat{M}$ is the Fourier transform ${ }^{1}$ of $M$, and $L_{\Phi^{\circ}}$ the Lebesgue measure on $\Phi^{\circ}$.

Now let us suppose that the continuous dual $V^{\prime}$ is endowed with a symplectic form $\sigma: V^{\prime} \times V^{\prime} \rightarrow \mathbb{R}$, i.e. an antisymmetric, non degenerate, and bilinear form. Then it is possible to construct in a unique fashion the Weyl $\mathrm{C}^{*}$-algebra of canonical commutation relations $\mathbf{W}\left(V^{\prime}, \sigma\right)$. Let us remark that we consider the Weyl $\mathrm{C}^{*}$-algebra to depend on a semiclassical parameter $h$, appearing in the so-called Weyl's relations among the generators $\left\{W(\xi), \xi \in V^{\prime}\right\}$ of the algebra:

$$
\forall \xi, \zeta \in V^{\prime}, W(\xi) W(\zeta)=e^{-i h \sigma(\xi, \zeta)} W(\xi+\zeta) .
$$

From time to time, to avoid confusion, the dependence on $h$ of $\mathbf{W}_{h}\left(V^{\prime}, \sigma\right)$ will be made explicit.

The regular states on $\mathbf{W}_{h}\left(V^{\prime}, \sigma\right)$ play an important role to study its semiclassical behavior $h \rightarrow 0$; their definition is recalled below.

Definition 2.2 (Regular states). A positive element $\omega \in \mathbf{W}\left(V^{\prime}, \sigma\right)^{\prime}$ of the continuous dual of the Weyl $C^{*}$-algebra is a regular state iff the $\mathbb{R}$-action

$$
t \mapsto \omega(W(t \xi))
$$

is continuous for any $\xi \in V^{\prime}$. A regular state is normalized iff $\omega(W(0))=1$.

Given any $\omega \in \mathbf{W}\left(V^{\prime}, \sigma\right)^{\prime}$, let us denote by $\left(H_{\omega}, \pi_{\omega}, \Omega_{\omega}\right)$ the corresponding GNS representation of $\mathbf{W}\left(V^{\prime}, \sigma\right)$. Let us recall that $H_{\omega}$ is a Hilbert space,

$$
\pi_{\omega}: \mathbf{W}\left(V^{\prime}, \sigma\right) \rightarrow \mathscr{B}\left(H_{\omega}\right)
$$

a *homomorphism, and $\Omega_{\omega} \in H_{\omega}$ the canonical cyclic vector of the representation. If $\omega$ is regular, it is possible to define - by Stone's theorem - the self-adjoint generator of the unitary group $\pi_{\omega}(W(\mathbb{R} \xi))$ for any $\xi \in V^{\prime}$. Let us denote such generator by

$$
\varphi_{\omega}(\xi)=\left.\frac{\mathrm{d} \pi_{\omega}(W(t \xi))}{\mathrm{d} t}\right|_{t=0} .
$$

For any finite dimensional symplectic subspace ${ }^{2} \Phi^{\circ} \subset V^{\prime}$, the operators $\varphi_{\omega}\left(\Phi^{\circ}\right)$ share a common dense set of analytic vectors [10, Lemma 5.2.12]. In order to introduce the well-known creation and annihilation operators $a_{\omega}^{*}\left(\Phi^{\circ}\right)$ and $a_{\omega}\left(\Phi^{\circ}\right)$, it is useful to see the symplectic $\Phi^{\circ}$ as a complex finite dimensional inner product space. Let us denote by $J_{\sigma}: \Phi^{\circ} \rightarrow \Phi^{\circ}, J_{\sigma}^{2}=-1$, a linear complex structure on $\Phi^{\circ}$

\footnotetext{
${ }^{1}$ We define the Fourier transform of a distribution both as an isometry on $L^{2}$ and an algebra homomorphism on $L^{1}$, i.e. $\hat{f}(\xi)=\int e^{-2 \pi i \xi \cdot x} f(x) \mathrm{d} x$. On the contrary we define, following common practice, the Fourier transform of a measure by $\hat{\mu}(\xi)=\int e^{i \xi(x)} \mathrm{d} \mu(x)$.

${ }^{2}$ If $V$ is locally convex, $F \subset V^{\prime}$ is a subspace of finite dimension iff there exists $\Phi \in F(V)$ such that $F=\Phi^{\circ}$. Therefore we adopt directly the notation $\Phi^{\circ}$ for finite dimensional subspaces of $V^{\prime}$.
} 
such that

$$
\sigma\left(J_{\sigma} \cdot, J_{\sigma} \cdot\right)=\sigma(\cdot, \cdot) \cdot
$$

Then $\Phi^{\circ}$ (of real dimension $2 n, n \in \mathbb{N}$ ) can be seen as a complex vector space (of complex dimension $n$ ) defining

$$
(a+i b) \xi=a \xi+b J_{\sigma} \xi
$$

and it is an inner product space by means of the Hermitian form

$$
\sigma\left(\cdot, J_{\sigma} \cdot\right)+i \sigma(\cdot, \cdot)
$$

The space $\Phi^{\circ}$ is the polar of some $\Phi \in F(V)$, and therefore

$$
V / \Phi \stackrel{\imath_{\sigma}}{\cong}(V / \Phi)^{\prime} \stackrel{{ }^{\mathrm{p}} p_{\Phi}}{\cong} \Phi^{\circ}
$$

The isomorphism $\imath_{\sigma}$ is chosen in a way such that for any $\xi \in \Phi^{\circ}$, and $w \in V / \Phi$,

$$
\sigma\left(\xi, J_{\sigma}{ }^{\mathrm{t}} p_{\Phi} \circ \imath_{\sigma}(w)\right)={ }^{\mathrm{t}} p_{\Phi}^{-1}(\xi)(w) .
$$

The creation and annihilation operators are then defined for any $\xi \in \Phi^{\circ}$ by

$$
\begin{aligned}
& a_{\omega}^{*}(\xi)=\frac{1}{2}\left(\varphi_{\omega}(\xi)-i \varphi_{\omega}(i \xi)\right), \\
& a_{\omega}(\xi)=\frac{1}{2}\left(\varphi_{\omega}(\xi)+i \varphi_{\omega}(i \xi)\right) .
\end{aligned}
$$

They are closed, and adjoint of each other when defined on $D\left(\varphi_{\omega}(\xi)\right) \cap D\left(\varphi_{\omega}(i \xi)\right)$. The self-adjoint number operator is a combination of the creation and annihilation operators along a (complex) orthonormal basis of $\Phi^{\circ}$. Let $\left\{\zeta_{j}\right\}_{j=1}^{n}$ be an o.n.b. of $\Phi^{\circ}$, then the number operator associated to $\Phi \in F(V)$ is defined by

$$
N_{\omega, \Phi}=\sum_{j=1}^{n} a_{\omega}^{*}\left(\zeta_{j}\right) a_{\omega}\left(\zeta_{j}\right) .
$$

Finally, let us define the scale of Hilbert spaces $\left(H_{\omega, \Phi}^{\delta}\right)_{\delta \in \mathbb{R}}$ associated to the number operator. For any $\delta \in \mathbb{R}^{+}, H_{\omega, \Phi}^{\delta}=D\left(N_{\omega, \Phi}^{\delta}\right)$ endowed with the norm

$$
\left\|\left\langle N_{\omega, \Phi}\right\rangle^{\delta} \cdot\right\|_{H_{\omega}}
$$

$H_{\omega, \Phi}^{-\delta}$ is the completion of $H_{\omega}$ with respect to the norm

$$
\left\|\left\langle N_{\omega, \Phi}\right\rangle^{-\delta} \cdot\right\|_{H_{\omega}} .
$$

Here $\langle\cdot\rangle$ stands for $\left(|\cdot|^{2}+1\right)^{\frac{1}{2}}$. The scale of spaces $\left(H_{\omega, \Phi}^{\delta}\right)_{\delta \in \mathbb{R}}$ satisfies

$$
\forall \delta \leq \delta^{\prime}, H_{\omega, \Phi}^{\delta} \subseteq H_{\omega, \Phi}^{\delta^{\prime}} .
$$


Let us denote by $\mathscr{S}_{\omega, \Phi}$ the nuclear space

$$
\mathscr{S}_{\omega, \Phi}=\bigcap_{\delta \in \mathbb{N}} H_{\omega, \Phi}^{\delta}
$$

and by $\mathscr{S}_{\omega, \Phi}^{\prime}$ its continuous dual

$$
\mathscr{S}_{\omega, \Phi}^{\prime}=\bigcup_{\delta \in \mathbb{N}} H_{\omega, \Phi}^{-\delta}
$$

The definitions above are natural to study the pseudodifferential calculus for cylindrical symbols on $V$ (with base $\Phi \in F(V)$ of codimension $2 n$ ), for it reduces to the standard finite-dimensional pseudodifferential calculus. In fact, the Weyl operators $\pi_{\omega}\left(W\left(\Phi^{\circ}\right)\right)$, and the creation and annihilation operators $a_{\omega}^{\#}\left(\Phi^{\circ}\right)$ are equivalent to their counterparts on the Schrödinger or Bargmann-Fock representation of the finite dimensional Weyl $\mathrm{C}^{*}$-algebra $\mathbf{W}\left(\Phi^{\circ}, \sigma\right)$; while $\mathscr{S}_{\omega, \Phi}$ plays the role of the Schwartz space $\mathscr{S}\left(\mathbb{R}^{n}\right)$, and $\mathscr{S}_{\omega, \Phi}^{\prime}$ of the tempered distributions $\mathscr{S}^{\prime}\left(\mathbb{R}^{n}\right)$. We assume that the reader is familiar with the techniques and results of pseudodifferential calculus and semiclassical analysis in finite dimensions [13, 16, 17, 24, are just a few of the monographs on the subject]. As a reference, in the remaining of this section we formulate, in the cylindrical setting, the results that we will use the most.

Definition 2.3 (Weyl quantization of a cylindrical symbol). Let $f$ be a cylindrical symbol on $V$ with base $\Phi \in F(V)$, such that $\hat{f}_{\Phi} \in L^{1}\left(\Phi^{\circ}\right)$. Then the Weyl quantization $\mathrm{Op}_{\frac{1}{2}}^{h}(f) \in \mathbf{W}\left(V^{\prime}, \sigma\right)$ of $f$ is defined by the Bochner integral

$$
\mathrm{Op}_{\frac{1}{2}}^{h}(f)=\int_{\Phi^{\circ}} \hat{f}_{\Phi}(\xi) W_{h}(2 \pi \xi) \mathrm{d} L_{\Phi \circ}(\xi) .
$$

On any regular representation $\left(H_{\omega}, \pi_{\omega}, \Omega_{\omega}\right)$ of $\mathbf{W}\left(V^{\prime}, \sigma\right)$, and for any $f_{\Phi} \in$ $\mathscr{S}^{\prime}(V / \Phi)$, the Weyl quantization $\pi_{\omega}\left(\mathrm{Op}_{\frac{1}{2}}^{h}(f)\right)$ is a continuous map from $\mathscr{S}_{\omega, \Phi}$ to $\mathscr{S}_{\omega, \Phi}^{\prime}$ defined by

$$
\forall \psi, \varphi \in \mathscr{S}_{\omega, \Phi},\left\langle\psi, \pi_{\omega}\left(\mathrm{Op}_{\frac{1}{2}}^{h}(f)\right) \varphi\right\rangle_{H_{\omega}}=\left(\hat{f}_{\Phi},\left\langle\psi, \pi_{\omega}\left(W_{h}(2 \pi \cdot)\right) \varphi\right\rangle_{H_{\omega}}\right)_{\mathscr{S}^{\prime} \times \mathscr{S}} .
$$

In Definition 2.3, we stressed the dependence on the semiclassical parameter $h$ of the Weyl quantizations (through the Weyl operators $W_{h}(2 \pi \xi)$ ). The semiclassical behavior of regular quantum states is obtained studying the corresponding generating functional. The generating functional of a state $\omega$ is defined for any $\xi \in V^{\prime}$ as

$$
G_{\omega}(\xi)=\omega(W(\xi))
$$

The generating functional defines uniquely regular states, i.e. there is a bijection between regular states and functions $G: V^{\prime} \rightarrow \mathbb{C}$ that are continuous when restricted to any finite dimensional subspace of $V$, and that are "almost" of positive 
type:

$$
\sum_{i, j \in F} G\left(\xi_{i}-\xi_{j}\right) e^{i h \sigma\left(\xi_{i}, \xi_{j}\right)} \bar{\lambda}_{j} \lambda_{i} \geq 0
$$

for any finite index set $F$, complex numbers $\left\{\lambda_{i}\right\}_{i \in F}$, and $\left\{\xi_{i}\right\}_{i \in F} \subset V^{\prime}[22]$. On the other hand, there is a bijection (the Fourier transform) between cylindrical measures on $V$ and functions $\Gamma: V^{\prime} \rightarrow \mathbb{C}$ that are continuous when restricted to any finite dimensional subspace of $V$, and of positive type:

$$
\sum_{i, j \in F} \Gamma\left(\xi_{i}-\xi_{j}\right) \bar{\lambda}_{j} \lambda_{i} \geq 0
$$

Therefore the topology of simple convergence on $\mathbb{C}^{V^{\prime}}$ induces a topology $\mathfrak{T}$ on the disjoint union of the sets of regular quantum states on $\mathbf{W}_{h}\left(V^{\prime}, \sigma\right), h>0$, together with the cylindrical measures on $V$ [see 12]. The Wigner or semiclassical measures associated to a generalized sequence $\left(\omega_{h_{\beta}}\right)_{\beta \in B}$ of regular states with no loss of mass are its $\mathfrak{T}$-cluster points as $h_{\beta} \rightarrow 0$ (more precisely, they are the $\mathfrak{P} \vee \mathfrak{T}$-cluster points, where $\mathfrak{P}$ is the topology induced by the ultraweak convergence of the finite dimensional measures, and $\mathfrak{P} \vee \mathfrak{T}$ is the upper bound topology). The "no loss of mass condition" is automatically satisfied if we assume either Hypothesis 3.2 or 3.6 .

Proposition $2.4([12])$. Let $\left(\omega_{h_{\gamma}}\right)_{\gamma \in C}, h_{\gamma} \rightarrow 0$, be a uniformly bounded net of regular states on $\left(\mathbf{W}_{h_{\gamma}}\left(V^{\prime}, \sigma\right)\right)_{\beta \in B}$ with no loss of mass. Then the set $\mathscr{W}\left(\omega_{h_{\gamma}}, \gamma \in C\right)$ of its $\mathfrak{T}$-cluster points is not empty, and each cluster point is a cylindrical measure on $V$, called a Wigner measure. Conversely, for any cylindrical measure $M$ on $V$ there is at least one net $\left(\omega_{h_{\gamma}}^{(M)}\right)_{\gamma \in C}$ of regular quantum states on $\left(\mathbf{W}_{h_{\gamma}}\left(V^{\prime}, \sigma\right)\right)_{\gamma \in C}$ that $\mathfrak{T}$-converges to it.

For any $\Phi \in F(V)$, we can define the topologies $\mathfrak{P}_{\Phi}$ and $\mathfrak{T}_{\Phi}$ on the restrictions to $\mathbf{W}\left(\Phi^{\circ}, \sigma\right)$ of regular quantum states on $\mathbf{W}\left(V^{\prime}, \sigma\right)$, together with the Radon measures on $V / \Phi$. We then have the following characterization of semiclassical measures.

\section{Lemma 2.5.}

$$
\left.\omega_{h_{n}}\right|_{\mathbf{W}_{h_{n}}\left(\Phi^{\circ}, \sigma\right)} \stackrel{h_{n} \rightarrow 0}{\longrightarrow} \mathfrak{P}_{\Phi} \mu_{\Phi}
$$

iff for any cylindrical symbol on $V$ with $f_{\Phi} \in C_{0}^{\infty}(V / \Phi)$

$$
\lim _{n \rightarrow \infty} \omega_{h_{n}}\left(\mathrm{Op}_{\frac{1}{2}}^{h_{n}}(f)\right)=\int_{V / \Phi} f_{\Phi}(w) \mathrm{d} \mu_{\Phi}(w) .
$$

If in addition there is no loss of mass, then

$$
\left.\omega_{h_{n}}\right|_{\mathbf{W}_{h_{n}}\left(\Phi^{\circ}, \sigma\right) \stackrel{h_{n} \rightarrow 0}{\longrightarrow} \mathfrak{P}_{\Phi} \vee \mathfrak{T}_{\Phi}} \mu_{\Phi}
$$


On any regular representation $\left(H_{\omega}, \pi_{\omega}, \Omega_{\omega}\right)$, it is possible to extend the only if part of Lemma 2.5 to polynomially bounded symbols. Let us denote by $\mathfrak{S}_{+}^{p}(H)$, $1 \leq p \leq \infty$, the cone of positive continuous operators on the Hilbert space $H$ that belong to the Schatten ideal of order $p$; and by $S_{\Phi}\left(\langle w\rangle_{\Phi}^{s}, g_{\Phi}\right), s \in \mathbb{R}$, the Hörmander class of symbols on $V / \Phi$, with the metric $g_{\Phi}$ to be either $|\mathrm{d} w|_{\Phi}^{2}$ or $\frac{|\mathrm{d} w|_{\Phi}^{2}}{\langle w\rangle_{\Phi}^{2}}$.

Proposition 2.6. Let $\Phi^{\circ} \subset V^{\prime}$ be a symplectic subspace. In addition, let $\left(\omega_{h_{\gamma}}\right)_{\gamma \in C}$ be a net of regular states on $\left(\mathbf{W}_{h_{\gamma}}\left(V^{\prime}, \sigma\right)\right)_{\gamma \in C}$, and $\varrho_{h_{\gamma}} \in \mathfrak{S}_{+}^{1}\left(H_{\omega}\right)$ for any $\gamma \in C$, such that for some $\delta \in \mathbb{R}^{+}$

$$
\operatorname{Tr}\left(\left\langle N_{\omega, \Phi}\right\rangle^{\delta} \varrho_{h_{\gamma}}\right)_{H_{\omega}} \leq C_{\delta}<\infty
$$

Then

$$
\varrho_{h_{\gamma}} \rightarrow \mathfrak{T} \mu=\left(\mu_{\Psi}\right)_{\Psi \in F(V)}
$$

implies that for any cylindrical $f$ on $V$ with base $\Phi$ and $f_{\Phi} \in S_{\Phi}\left(\langle w\rangle^{\delta}, g_{\Phi}\right)$,

$$
\lim _{\gamma \in C} \operatorname{Tr}\left(\pi_{\omega}\left(\mathrm{Op}_{\frac{1}{2}}^{h_{\gamma}}(f)\right) \varrho_{h_{\gamma}}\right)=\int_{V / \Phi} f_{\Phi}(w) \mathrm{d} \mu_{\Phi}(w)
$$

\section{Concentration of Wigner measures}

As explained in Section 2, to any bounded sequence $\left(\omega_{h_{\gamma}}\right)_{\gamma \in C}, h_{\gamma} \rightarrow 0$, of regular states on $\left(\mathbf{W}_{h_{\gamma}}\left(V^{\prime}, \sigma\right)\right)_{\gamma \in C}$ there corresponds a nonempty set of cylindrical measures on $V$, called Wigner measures. In applications, it is desirable to have sufficient conditions on $\left(\omega_{h_{\gamma}}\right)_{\gamma \in C}$ such that each corresponding Wigner measure is concentrated as a true Radon measure on some given topological vector space $W$ (that may or may not differ from $V$ ). The main purpose of this note is to provide such sufficient conditions, exploiting cylindrical semiclassical analysis. Any cylindrical measure $M=\left(\mu_{\Phi}\right)_{\Phi \in F(V)}$ on a locally convex space $V$ is a Borel Radon measure on the space

$$
\bar{V}=\prod_{\Phi \in F(V)} \overline{V / \Phi},
$$

product of Čech compactifications of the finite dimensional quotients, endowed with the product topology. The cylindrical measure $M \in \mathcal{M}_{\text {rad }}(\bar{V})$ concentrates as the Borel Radon measure $\mu \in \mathcal{M}_{\text {rad }}(X)$ on the real topological vector space $X$ iff there exists an injection $\varphi: X \rightarrow \bar{V}$,

$$
M(\bar{V} \backslash \varphi X)=0,
$$

and $M(b)=\mu\left(\varphi^{-1} b\right)$ for any Borel set $b \in \mathscr{B}(\bar{V}) \cap \varphi X$. If $X$ is a complex topological vector space, the injection is $\varphi: \mathbb{F} X \rightarrow \bar{V}$, where $\mathbb{F}$ is the forgetful 
functor from the category of complex vector spaces to the category of real vector spaces. The rest of the definition is modified accordingly.

3.1. Separable Hilbert spaces. Let $X$ be a complex vector space, and

$$
t: X \times X \rightarrow \mathbb{C}
$$

a sesquilinear form on $X$. Let

$$
q(x)=t(x, x)
$$

be the corresponding quadratic form. Then $t$ factors through $X / \operatorname{ker} q$, i.e. there is a sesquilinear form $t_{q}:(X / \operatorname{ker} q) \times(X / \operatorname{ker} q) \rightarrow \mathbb{C}$ such that

$$
t_{q}\left([x]_{q},[y]_{q}\right)=t(x, y) .
$$

With an abuse of notation, let us still denote $t_{q}$ by $t$. The completion $X_{q}$ of $X / \operatorname{ker} q$ with respect to the norm $\sqrt{q}$ is a complex Hilbert space with inner product $t$. Throughout this section, we suppose that $X_{q}$ is separable. The results of this section can be easily adapted to real vector spaces.

Let us denote by $\mathbb{F}$ the forgetful functor from the category of complex vector spaces to the category of real vector spaces. Therefore $\mathbb{F} X_{q}$ is a real Hilbert space with inner product Ret, and a symplectic space with symplectic form $\operatorname{Im} t$. In addition, if $\left\{x_{1}, \ldots, x_{n}, \ldots\right\}$ is a complex orthonormal basis of the space $X_{q}$, then $\left\{\mathbb{F} x_{1}, \mathbb{F}\left(i x_{1}\right), \ldots, \mathbb{F} x_{n}, \mathbb{F}\left(i x_{n}\right), \ldots\right\}$ is a real orthonormal basis of the space $\mathbb{F} X_{q}$. We make the following assumption of compatibility between the inner product of $X$ and the symplectic form $\sigma$ of $V^{\prime}$, dual to a locally convex space $V$ as in Section 2.

Hypothesis 3.1. $\mathbb{F} X \subseteq V^{\prime}$. In addition:

- The complex structure $J: \mathbb{F} X \rightarrow \mathbb{F} X$, defined by $J \mathbb{F} x=\mathbb{F}(i x)$, is such that

$$
\sigma(J \cdot J \cdot)=\sigma(\cdot, \cdot) .
$$

- There exists a linear map $\mathbf{m}: X \rightarrow X$ such that for any $x, y \in X$,

$$
t(x, y)=\sigma(\mathbb{F} \mathbf{m}(x), \mathbb{F} \mathbf{m}(i y))+i \sigma(\mathbb{F} \mathbf{m}(x), \mathbb{F} \mathbf{m}(y)) .
$$

Now let

$$
\left\{\left[e_{n}\right]_{q}, n \in \mathbb{N}\right\} \subset X / \operatorname{ker} q
$$

be an orthonormal basis of $X_{q}$. In addition, let

$$
\left\{\varepsilon_{n}=\mathbb{F} \mathbf{m}\left(e_{n}\right) ; n \in \mathbb{N}\right\} \subset V^{\prime} .
$$


Let us consider any finite-dimensional subspace of $X$ of the form

$$
E_{R}=\operatorname{span}_{\mathbb{C}}\left\{e_{0}, e_{1}, \ldots, e_{R}\right\}, R \in \mathbb{N} .
$$

The complex Hilbert space $E_{R}$ is isomorphic to $p_{R} X_{q}$, where

$$
p_{R}=\sum_{j=1}^{R} p\left(\left[e_{j}\right]_{q}\right)
$$

is the sum of rank one orthogonal projections. We define the symplectic subspace $\Phi_{E_{R}}^{\circ} \subset V^{\prime}$ as

$$
\Phi_{E_{R}}^{\circ}=\operatorname{span}_{\mathbb{R}}\left\{\varepsilon_{0}, J \varepsilon_{0}, \ldots, \varepsilon_{R}, J \varepsilon_{R}\right\} .
$$

As in Section 2, we remark that $\Phi_{E_{R}}^{\circ}$ can be seen as a complex inner product space with inner product

$$
b(\cdot, \cdot)=\sigma(\cdot, J \cdot)+i \sigma(\cdot, \cdot)
$$

By construction,

$$
\left(\Phi_{E_{R}}^{\circ}, b\right) \stackrel{i_{t}}{\cong}\left(E_{R}, t\right),
$$

as complex Hilbert spaces. Now let $\left(H_{\omega}, \pi_{\omega}, \Omega_{\omega}\right)$ be the GNS representation of $\mathbf{W}\left(V^{\prime}, \sigma\right)$ corresponding to a regular state $\omega$. We define the $\mathbf{m}$-creation and annihilation operators as follows. For any $x \in X$,

$$
\begin{aligned}
& \mathbf{a}_{\omega}^{*}(x)=\frac{1}{2}\left(\varphi_{\omega}(\mathbb{F} \mathbf{m}(x))-i \varphi_{\omega}(\mathbb{F} \mathbf{m}(i x))\right), \\
& \mathbf{a}_{\omega}(x)=\frac{1}{2}\left(\varphi_{\omega}(\mathbb{F} \mathbf{m}(x))+i \varphi_{\omega}(\mathbb{F} \mathbf{m}(i x))\right) .
\end{aligned}
$$

The corresponding $\mathbf{m}$-number operator on $\Phi_{E_{R}}^{\circ}$ is the generalization of the number operator to $\mathbf{m}$-creation and annihilation:

$$
\mathbf{N}_{\omega, R}=\sum_{j=1}^{R} \mathbf{a}_{\omega}^{*}\left(\mathbf{m}\left(e_{j}\right)\right) \mathbf{a}_{\omega}\left(\mathbf{m}\left(e_{j}\right)\right)
$$

For any $\delta>0$, the sequence $\left(\mathbf{N}_{\omega, R}^{\delta}\right)_{R \in \mathbb{N}}$ is a monotonically increasing sequence of positive self adjoint operators. If we denote by $\left(\mathbf{n}_{\omega, R}^{\delta}\right)_{R \in \mathbb{N}}$ the sequence of corresponding quadratic forms, then

$$
\mathbf{n}_{\omega, \infty}^{\delta}=\sup _{R \in \mathbb{N}} \mathbf{n}_{\omega, R}^{\delta}
$$

is a closed positive quadratic form on $H_{\omega}$ (not necessarily densely defined), with domain of definition

$$
D\left(\mathbf{n}_{\omega, \infty}^{\delta}\right)=\left\{\psi \in \bigcap_{R \in \mathbb{N}} D\left(\mathbf{n}_{\omega, R}^{\delta}\right), \sup _{R \in \mathbb{N}} \mathbf{n}_{\omega, R}^{\delta}(\psi)<\infty\right\} .
$$

Now let $\left(\psi_{h_{\gamma}}\right)_{\gamma \in C}, h_{\gamma} \rightarrow 0$, be a net of vectors in $H_{\omega_{h_{\gamma}}}$ for any $\gamma \in C$, and denote by $\mathscr{W}\left(\psi_{h_{\gamma}}, \gamma \in C\right)$ the set of Wigner cylindrical measures on $V$ associated to it 
- any vector in $H_{\omega}$ has an associated rank-one orthogonal projection that is a regular state on $\mathbf{W}\left(V^{\prime}, \sigma\right)$. The aim is to prove that the following condition is sufficient to prove that any $M \in \mathscr{W}\left(\psi_{h_{\gamma}}, \gamma \in C\right)$ concentrates as a (finite) Borel Radon measure $\mu$ on the Hilbert space $X_{q}$.

Hypothesis 3.2. Let $\left(\psi_{h_{\gamma}}\right)_{\gamma \in C}$ be a net of vectors on regular GNS representations of $\mathbf{W}_{h_{\gamma}}\left(V^{\prime}, \sigma\right)$, with

$$
\sup _{\gamma \in C}\left\|\psi_{h_{\gamma}}\right\|_{H_{\omega_{h \gamma}}}<\infty .
$$

There exists $\delta>0$ such that $\psi_{h_{\gamma}} \in D\left(\mathbf{n}_{\omega_{h_{\gamma}}, \infty}\right)$ for any $\gamma \in C$, and there exists $\mathcal{K}>0$ such that uniformly in $\gamma \in C$,

$$
\mathbf{n}_{\omega, \infty}^{\delta}\left(\psi_{h_{\gamma}}\right) \leq \mathcal{K}
$$

First of all, any $M \in \mathscr{W}\left(\psi_{h_{\gamma}}, \gamma \in C\right)$ is a cylindrical measure on $V$. Therefore $M=\left(\mu_{\Phi}\right)_{\Phi \in F(V)}$. Now let us consider $\left(\mu_{\Phi_{R}}\right)_{R \in \mathbb{N}} \subset\left(\mu_{\Phi}\right)_{\Phi \in F(V)}$, where $\Phi_{R}=\Phi_{E_{R}}^{\circ \circ}$ is the polar of $\Phi_{E_{R}}^{\circ}$. $\Phi_{R}$ belongs to $F(V)$, and satisfies

$$
\Phi_{R}^{\circ}=\Phi_{E_{R}}^{\circ \circ \circ}=\Phi_{E_{R}}^{\circ},
$$

via the bipolar theorem [9]. Therefore by means of the isomorphisms (see Section 2)

$$
V / \Phi_{R} \stackrel{\imath_{\sigma}}{\cong}\left(V / \Phi_{R}\right)^{\prime} \stackrel{{ }^{\mathrm{t}} p_{\Phi_{R}}}{\cong} \Phi_{R}^{\circ} \stackrel{i_{t}}{\cong} E_{R}
$$

it is possible to identify each $V / \Phi_{R}$ with $E_{R}$, i.e. with a complex finite dimensional Hilbert subspace of $X_{q}$. Using the aforementioned identification, it follows that

$$
\forall R \in \mathbb{N}, V / \Phi_{R} \subset V / \Phi_{R+1} ; \bigcup_{R \in \mathbb{N}} V / \Phi_{R}=X_{q}
$$

Finally, for any $j \in \mathbb{N}$ the orthogonal projections

$$
p_{R+j, R}: E_{R+j} \rightarrow E_{R}
$$

coincide with the projections

$$
p_{\Phi_{R+j}, \Phi_{R}}: V / \Phi_{R+j} \rightarrow V / \Phi_{R}
$$

relative to the projective family $M=\left(\mu_{\Phi}\right)_{\Phi \in F(V)}$. Therefore $\left(\mu_{\Phi_{R}}\right)_{R \in \mathbb{N}}$ is a projective subfamily of $M$. As proved in [23], $M$ is concentrated as a Borel Radon measure $\mu$ on $X_{q}$ iff

$$
\forall R \in \mathbb{N}, \quad \lim _{r \rightarrow \infty} \mu_{\Phi_{R}}\left(\left\{f \in V / \Phi_{R}, q(f) \geq r\right\}\right)=0,
$$

where $q$ is the Euclidean norm on $V / \Phi_{R}$ (seen as a complex space), that coincides with the Hilbert norm of $X_{q}$. In fact, if we denote by

$$
\mu_{E_{R}}=i_{t *}{ }^{\mathrm{t}} p_{\Phi_{R} *} \imath_{\sigma *} \mu_{\Phi_{R}}
$$


the pushforward image of $\mu_{\Phi_{R}},(14)$ is equivalent to

$$
\lim _{r \rightarrow \infty} \mu_{E_{R}}\left(\left\{f \in E_{M}, q(f) \geq r\right\}\right)=0 .
$$

Suppose now that we have proved that $M$ concentrates as a Radon measure $\mu$ on $X_{q}$. In addition, let the following condition be satisfied:

$$
\exists \delta>0, \exists \mathcal{K}>0, \quad \int_{X_{q}} q(f)^{\delta} \mathrm{d} \mu(f) \leq \mathcal{K} .
$$

Equation (16) implies that $\hat{\mu}: X_{q} \rightarrow \mathbb{C}$ is continuous. Let $0<\delta \leq 1$, and let $g_{1}, g_{2} \in X_{q}$. Then for some $\mathcal{K}_{\delta}>0$

$$
\begin{array}{r}
\left|\hat{\mu}\left(g_{1}\right)-\hat{\mu}\left(g_{2}\right)\right| \leq \int_{X_{q}}\left|e^{i \operatorname{Re} t\left(g_{1}-g_{2}, f\right)}-1\right| \mathrm{d} \mu(f) \leq \mathcal{K}_{\delta} \int_{X_{q}}\left|t\left(g_{1}-g_{2}, f\right)\right|^{\delta} \mathrm{d} \mu(f) \\
\leq \mathcal{K}_{\delta} q\left(g_{1}-g_{2}\right)^{\delta} \int_{X_{q}} q(f)^{\delta} \mathrm{d} \mu(f) \leq \mathcal{K}_{\delta} \mathcal{K} q\left(g_{1}-g_{2}\right)^{\delta} .
\end{array}
$$

If $\delta>1$, then

$$
\begin{array}{r}
\int_{X_{q}} q(f) \mathrm{d} \mu(f)=\int_{X_{q}} 1_{\{q(\cdot) \leq 1\}}(f) q(f) \mathrm{d} \mu(f)+\int_{X_{q}} 1_{\{q(\cdot)>1\}}(f) q(f) \mathrm{d} \mu(f) \\
\leq \mu\left(X_{q}\right)+\mathcal{K},
\end{array}
$$

and therefore the result above for $\delta=1$ applies with constant $\mu\left(X_{q}\right)+\mathcal{K}$.

Theorem 3.3. Let $V$ be a locally convex space with dual $\left(V^{\prime}, \sigma\right)$ endowed with a symplectic form. In addition, let $X$ be a complex vector space with a sesquilinear form $t$ such that the compatibility condition 3.1 with $\sigma$ holds.

For a cylindrical measure $M \in \mathscr{W}\left(\psi_{h_{\gamma}}, \gamma \in C\right)$ to be concentrated as a Borel Radon measure $\mu$ on the separable Hilbert space $X_{q}$, it is sufficient that Hypothesis 3.2 is satisfied. The Fourier transform $\hat{\mu}$ is furthermore continuous on $X_{q}$; and if $\tilde{\delta}>0$ and $\tilde{\mathcal{K}}>0$ are the values for which 3.2 holds, then

$$
\int_{X_{q}} q(f)^{\tilde{\delta}} \mathrm{d} \mu(f) \leq \tilde{\mathcal{K}} .
$$

Proof. Hypothesis 3.2 is sufficient to have no loss of mass (and hence convergence of the generating functional) when the state is restricted to each $\Phi_{R}^{\circ}$. We have to prove that Hypothesis 3.2 also implies both (14) and (16). First of all, let us prove (14). As discussed in Section 1.1, the m-creation and annihilation operators establish a correspondence with the standard pseudodifferential calculus associated to the Heisenberg group $\mathbf{H}\left(\Phi_{E_{R}}^{\circ}, \sigma\right)$, and that is assured by the compatibility condition 3.1. Then (14) is proved using the argument introduced in [3, Theorem 6.2]. Let $\left(\psi_{h_{\gamma}}\right)_{\gamma \in C}$ be a net of vectors in regular GNS representations, and let 
$\Phi_{E_{R}}^{\circ}$ be defined as above for some $R \in \mathbb{N}$. Then Hypothesis 3.2 yields that

$$
\left\langle\psi_{h_{\gamma}}, \mathbf{N}_{\omega_{h_{\gamma}}, R}^{\tilde{\delta}} \psi_{h_{\gamma}}\right\rangle_{H_{\omega_{h \gamma}}} \leq \tilde{\mathcal{K}}
$$

uniformly in $\gamma$ for some $\tilde{\delta}>0$. By means of the link between Wick and Weyl quantization, it is well-known that

$$
1+\frac{h R}{2}+\mathbf{N}_{\omega_{h}, R}=\mathbf{O p}_{\frac{1}{2}}^{h}(1+q(\cdot))
$$

where $q: V / \Phi_{R} \rightarrow \mathbb{R}^{+}$is the euclidean squared norm of the finite dimensional (complex) Hilbert space $V / \Phi_{R} \cong E_{R}$, and $\mathbf{O p}_{\frac{1}{2}}^{h}$ is the Weyl quantization procedure defined in Section 2, but with each $W_{h}(\mathbb{F} x), x \in X$, replaced by $W_{h}(\mathbb{F} \mathbf{m}(x))$. The operator

$$
\mathbf{O p}_{\frac{1}{2}}^{h}(1+q(\cdot))
$$

is fully elliptic [see e.g. 15]. Therefore for any $s \in \mathbb{R}$,

$$
\left(1+\frac{h R}{2}+\mathbf{N}_{\omega_{h}, R}\right)^{s}=\mathbf{O p}_{\frac{1}{2}}^{h}\left((1+q(\cdot))^{s}+O(h)\right),
$$

where the function $h^{-1} O(h)$ is uniformly bounded in the Hörmander class

$$
S_{R}\left(\langle w\rangle_{\Phi_{R}}^{s-2}, \frac{|\mathrm{d} w|_{\Phi_{R}}^{2}}{\langle w\rangle_{\Phi_{R}}^{2}}\right)
$$

For any $h_{\gamma} \leq R^{-1},(17)$ implies there exists $\tilde{\mathcal{K}}_{\tilde{\delta}}>0$ such that

$$
\left\langle\psi_{h_{\gamma}},\left(1+\frac{h_{\gamma} R}{2}+\mathbf{N}_{\omega_{h_{\gamma}}, R}\right)^{\tilde{\delta}} \psi_{h_{\gamma}}\right\rangle_{H_{\omega_{h_{\gamma}}}} \leq \tilde{\mathcal{K}}_{\tilde{\delta}}
$$

Now let $r \geq 1$, and $C_{R} \subseteq C$ such that for any $\gamma \in C_{R}, h_{\gamma} \leq R^{-1}$. Using standard pseudodifferential techniques in $V / \Phi_{R}$ [see 3], it is possible to prove that for any $\gamma \in C_{R}$ and $\delta>0$, there exists a $K>0$ such that the following operator inequality holds on $H_{\omega_{h \gamma}}$ :

$$
\mathbf{O p}_{\frac{1}{2}}^{h_{\gamma}}\left(\chi\left(r^{-\frac{1}{2}} \cdot\right)\right) \leq(1+r)^{-\delta}\left(1+2 K h_{\gamma}\right) \mathbf{O p}_{\frac{1}{2}}^{h_{\gamma}}\left((1+q(\cdot))^{-\delta / 2}\right)^{-2} .
$$

Here $\chi(\cdot) \in C^{\infty}\left(V / \Phi_{R}\right)$ is non-negative and such that $\chi=0$ in a neighbourhood of $\left\{f \in V / \Phi_{R}, \sqrt{q(f)} \leq 1\right\}$. In addition, (18) yields there exists $K^{\prime}>0$ such that

$$
\left\|\mathbf{O p}_{\frac{1}{2}}^{h_{\gamma}}\left((1+q(\cdot))^{-\delta / 2}\right)^{-2}\left(1+\frac{h_{\gamma} R}{2}+\mathbf{N}_{\omega_{h_{\gamma}}, R}\right)^{\delta}\right\|_{\mathscr{B}\left(H_{\omega_{h \gamma}}\right)} \leq K^{\prime} h_{\gamma}+1 .
$$

Therefore for any $\gamma \in C_{M}$ and $r \geq 1$, there exists $\tilde{K}_{\tilde{\delta}}>0$ such that

$$
\left\langle\psi_{h_{\gamma}}, \mathbf{O p}_{\frac{1}{2}}^{h_{\gamma}}\left(\chi\left(r^{-\frac{1}{2}} \cdot\right)\right) \psi_{h_{\gamma}}\right\rangle_{H_{\omega_{h}}} \leq \tilde{K}_{\tilde{\delta}}(1+r)^{-\tilde{\delta}}
$$

Now let $M \in \mathscr{W}\left(\psi_{h_{\gamma}}, \gamma \in C\right)$, and $\left(\psi_{h_{\gamma}}\right)_{\gamma \in \underline{C}}$ the subnet of $\left(\psi_{h_{\gamma}}\right)_{\gamma \in C_{M}}$ whose restrictions to $\left(\mathbf{W}_{h_{\gamma}}\left(\Phi_{R}^{\circ}, \sigma\right)\right)_{\gamma \in C_{M}}, R \in \mathbb{N}$, converge to $\mu_{\Phi_{R}}$ with respect to the 
$\mathfrak{T}_{\Phi_{R}}$ topology. Then by Proposition 2.6,

$$
\lim _{\gamma \in \underline{C}}\left\langle\psi_{h_{\gamma}}, \mathbf{O p}_{\frac{1}{2}}^{h_{\gamma}}\left(\chi\left(r^{-\frac{1}{2}} \cdot\right)\right) \psi_{h_{\gamma}}\right\rangle_{H_{\omega_{h_{\gamma}}}}=\int_{V / \Phi_{R}} \chi\left(r^{-\frac{1}{2}} f\right) \mathrm{d} \mu_{\Phi_{R}}(f) .
$$

Hence it follows that

$$
\begin{aligned}
& \mu_{\Phi_{R}}\left(\left\{f \in V / \Phi_{R}, q(f) \geq r\right\}\right) \leq \int_{V / \Phi_{R}} \chi\left(r^{-\frac{1}{2}} f\right) \mathrm{d} \mu_{\Phi_{R}}(f) \\
& =\lim _{\gamma \in \underline{C}}\left\langle\psi_{h_{\gamma}}, \mathbf{O p}_{\frac{1}{2}}^{h_{\gamma}}\left(\chi\left(r^{-\frac{1}{2}} \cdot\right)\right) \psi_{h_{\gamma}}\right\rangle_{H_{\omega_{h_{\gamma}}}} \leq \tilde{K}_{\tilde{\delta}}(1+r)^{-\tilde{\delta}} .
\end{aligned}
$$

It remains to prove (16) for $\tilde{\delta}$ and $\tilde{\mathcal{K}}$. Let $\mathbf{1}_{0} \in C_{0}^{\infty}\left(V / \Phi_{R}\right)$ be a smooth indicator function of a neighbourhood of 0 . The following inequality, for any $\delta>0$ and $r \geq 1$, and some $\mathcal{K}_{R}>0$, is again a consequence of standard pseudodifferential techniques:

$$
\left(\mathbf{N}_{\omega_{h}, R}\right)^{\delta} \geq \mathbf{O} \mathbf{p}_{\frac{1}{2}}^{h}\left(q(\cdot)^{\delta} \mathbf{1}_{0}\left(r^{-1} \cdot\right)\right)-\mathcal{K}_{R} h\left(\mathbf{N}_{\omega_{h}, R}\right)^{\delta}
$$

Reasoning as above, and using the fact that by (14) $M$ concentrates as a Radon measure $\mu$ on $X_{q}$, we obtain

$$
\int_{E_{R}} q(f)^{\tilde{\delta}} \mathrm{d} \mu(f) \leq \tilde{\mathcal{K}} .
$$

Now since the bound is uniform with respect to $R \in \mathbb{N}$, we can take the limit $R \rightarrow \infty$ using dominated convergence.

3.2. Duals of nuclear spaces. Now, let us suppose that $V^{\prime} \supset \mathscr{N}$, where $\mathscr{N}$ is a nuclear barrelled space. Then concentration of Wigner measures on $\mathscr{N}^{\prime}$, endowed with any topology $\mathfrak{R}$ between $\sigma\left(\mathscr{N}^{\prime}, \mathscr{N}\right)$ and the Mackey topology $\tau\left(\mathscr{N}^{\prime}, \mathscr{N}\right)$, admits an easy characterization.

It is well-known that a cylindrical measure $M$ on $V$ is concentrated as a Radon measure $\mu$ on $\mathscr{N}_{\mathfrak{R}}^{\prime}$ iff $\left(\mu_{\Phi}\right)_{\Phi \in F\left(\mathscr{N}_{\mathfrak{R}}^{\prime}\right)}$ can be identified with a projective subfamily of $M$ and the restriction of $\hat{M}$ to $\mathscr{N}$ is continuous [see e.g. 8]. Continuity properties of the Fourier transform of $M$ are inherited by continuity properties of the family of generating functionals of regular states $\omega_{h_{\gamma}}, \gamma \in C$. In particular, equicontinuity of

$$
\mathbf{G}_{B}=\left\{\left.G_{\omega_{h_{\gamma}}}\right|_{\mathscr{N}}, \gamma \in C\right\}
$$

is sufficient to guarantee the continuity of its cluster points (in the topology of simple convergence), as $h_{\gamma} \rightarrow 0$. This is in turn sufficient to prove that any $M \in \mathscr{W}\left(\omega_{h_{\gamma}}, \gamma \in C\right)$ is concentrated as a Radon measure on $\mathscr{N}_{\mathfrak{R}}^{\prime}$.

Theorem 3.4. Let $\left(\omega_{h_{\gamma}}\right)_{\gamma \in C}$ be a net of regular states on $\mathbf{W}_{h_{\gamma}}\left(V^{\prime}, \sigma\right)$ such that

$$
\sup _{\gamma \in C} \omega_{h_{\gamma}}\left(W_{h_{\gamma}}(0)\right)<\infty \text {. }
$$


If there exists a nuclear barrelled space $\mathscr{N}$ such that $\mathscr{N} \subseteq V^{\prime}$, and $(V / \Phi)_{\Phi \in F\left(\mathscr{N}_{\mathfrak{R}}^{\prime}\right)}$ is identified with a projective subfamily of $(V / \Phi)_{\Phi \in F(V)}$, then equicontinuity of the family $\mathbf{G}_{B}$ defined in (19) implies that any $M \in \mathscr{W}\left(\omega_{h_{\gamma}}, \gamma \in C\right)$ is concentrated as a Borel Radon measure $\mu$ on $\mathscr{N}_{\mathfrak{R}}^{\prime}$.

The formulation of Theorem 3.4 seems quite natural, however it may be difficult to apply it in concrete situations, for the equicontinuity of $\mathbf{G}_{B}$ may be hard to prove without a detailed knowledge of the generating functional.

3.3. Duals of Banach spaces. In this section we consider concentration in dual spaces $\mathcal{X}^{\prime}$, where $\mathcal{X}$ is a Banach space. There is no loss of generality in considering $\mathcal{X}$ to be real, and all the results extend to complex Banach spaces. It should also be possible to formulate a similar result for duals of locally convex spaces, using the Bourbaki-Alaoglu variant of the Banach-Alaoglu's Theorem.

The idea is to adapt the procedure used in Section 3.1 to prove tightness in $\mathcal{X}^{\prime}$ of a suitable projective subfamily of $M \in \mathscr{W}\left(\psi_{h_{\gamma}}, \gamma \in C\right)$. However, we will only be able to prove the concentration of $M$ as a Borel Radon measure $\mu$ on $\mathcal{X}^{\prime}$ endowed with the ultraweak dual topology. To avoid confusion, let us denote by $\mathcal{X}_{\mathrm{w}}^{\prime}$ the space $\mathcal{X}^{\prime}$ endowed with the $\sigma\left(\mathcal{X}^{\prime}, \mathcal{X}\right)$ topology. Let us recall that $\left(\mathcal{X}_{\mathrm{w}}^{\prime}\right)^{\prime}=\mathcal{X}$.

Lemma 3.5 (Tightness). A cylindrical measure $\left(\mu_{\Psi}\right)_{\Psi \in F\left(B_{\mathrm{w}}^{\prime}\right)}$ is concentrated as a Borel Radon measure $\mu$ on $\mathcal{X}_{\mathrm{w}}^{\prime}$ if for any $\varepsilon>0$, there exists a $\delta>0$ such that for any $\Psi \in F\left(\mathcal{X}_{\mathrm{w}}^{\prime}\right)$ of codimension $2 n, n \in \mathbb{N}$,

$$
\mu_{\Psi}\left(\mathcal{X}_{\mathrm{w}}^{\prime} / \Psi \backslash p_{\Psi}\left(\left\{x \in \mathcal{X}^{\prime},\|x\|_{\mathcal{X}^{\prime}}^{2} \leq \delta\right\}\right)\right)<\varepsilon .
$$

Proof. By Banach-Alaoglu's theorem the ball $\left\{x \in \mathcal{X}^{\prime},\|x\|_{\mathcal{X}^{\prime}}^{2} \leq \delta\right\}$ is compact in $\mathcal{X}_{\mathrm{w}}^{\prime}$. In addition, the projective family of measures indexed by spaces of even codimension is a projective subfamily of $\left(\mu_{\Psi}\right)_{\Psi \in F\left(\mathcal{X}_{\mathrm{w}}^{\prime}\right)}$ with cofinal index set. By a theorem of Prokhorov [21, Theorem I.21], (20) is then sufficient to prove that $\mu$ is concentrated as a Radon measure on $\mathcal{X}_{\mathrm{w}}^{\prime}$.

The sufficient condition on quantum states is similar to the one given for separable Hilbert spaces.

Hypothesis 3.6. Let $\left(\psi_{h_{\gamma}}\right)_{\gamma \in C}$ be a net of vectors on regular GNS representations of $\mathbf{W}_{h_{\gamma}}(\mathcal{X}, \sigma)$, with

$$
\sup _{\gamma \in C}\left\|\psi_{h_{\beta}}\right\|_{H_{\omega_{h \gamma}}}<\infty
$$

There exists $\delta>0$ and $\mathcal{K}>0$ such that uniformly in $\gamma \in C$,

$$
\sup _{\substack{\Psi \in F\left(\mathcal{X}_{\mathrm{w}}^{\prime}\right) \\ \operatorname{dim} \mathcal{X}_{\mathrm{w}}^{\prime} / \Psi=2 n}}\left\langle\psi_{h_{\gamma}}, N_{\omega_{h_{\gamma}}, \Psi}^{\delta} \psi_{h_{\gamma}}\right\rangle \leq \mathcal{K} .
$$


The strategy is the same as the one used to prove (14) in Theorem 3.3. Let $K=\left\{x \in \mathcal{X},\|x\|_{\mathcal{X}}^{2} \leq 1\right\}$. The key point is to identify, for any $\Psi$ with even codimension, $K \cap \Psi^{\circ}$ with $p_{\Psi}\left(K^{\circ}\right)^{\circ}$. It is well-known that

$$
K^{\circ}=\left\{\xi \in \mathcal{X}^{\prime},\|\xi\|_{\mathcal{X}^{\prime}}^{2} \leq 1\right\}
$$

In addition, $K \cap \Psi^{\circ}$ is a closed bounded absorbing absolutely convex neighbourhood of zero, and therefore a closed ball centered in zero (in the locally compact topology of the finite dimensional real vector space $\left.\Psi^{\circ}\right)$. It is possible to make the identification $\mathcal{X}_{\mathrm{w}}^{\prime} / \Psi \stackrel{\jmath}{\cong}\left(\mathcal{X}_{\mathrm{w}}^{\prime} / \Psi\right)^{\prime}$ in a way such that for any $\xi \in\left(\mathcal{X}_{\mathrm{w}}^{\prime} / \Psi\right)^{\prime}$ and $[x] \in \mathcal{X}_{\mathrm{w}}^{\prime} / \Psi$

$$
\jmath^{-1}(\xi) \cdot[x]=\xi([x])=\xi \cdot \jmath([x]) .
$$

Lemma 3.7. Let $\left(\mathcal{X}_{\mathrm{w}}^{\prime} / \Psi\right)^{\prime} \stackrel{{ }^{\mathrm{p}}{ }_{\Psi}}{\cong} \Psi^{\circ}$ be the homeomorphism that yields, for any $x \in \mathcal{X}_{\mathrm{w}}^{\prime}$ and $\xi \in\left(\mathcal{X}_{\mathrm{w}}^{\prime} / \Psi\right)^{\prime}$,

$$
\xi([x])={ }^{\mathrm{t}} p_{\Psi}(\xi)(x) .
$$

Then

$$
K \cap \Psi^{\circ}={ }^{\mathrm{t}} p_{\Psi}\left(p_{\Psi}\left(K^{\circ}\right)^{\circ}\right)
$$

Proof. By definition,

$$
p_{\Psi}\left(K^{\circ}\right)^{\circ}=\left\{\xi \in\left(\mathcal{X}_{\mathrm{w}}^{\prime} / \Psi\right)^{\prime}, \forall x \in K^{\circ}|\xi([x])| \leq 1\right\} .
$$

Applying the isomorphism, it follows by (22) that

$$
\begin{array}{r}
{ }^{\mathrm{t}} p_{\Psi}\left(p_{\Psi}\left(K^{\circ}\right)^{\circ}\right)=\left\{{ }^{\mathrm{t}} p_{\Psi}(\xi) \in{ }^{\mathrm{t}} p_{\Psi}\left(\left(\mathcal{X}_{\mathrm{w}}^{\prime} / \Psi\right)^{\prime}\right), \forall x \in K\left|{ }^{\mathrm{t}} p_{\Psi}(\xi)(x)\right| \leq 1\right\} \\
=\left\{\zeta \in \Psi^{\circ}, \forall x \in K^{\circ}|\zeta(x)| \leq 1\right\}=K^{\circ \circ} \cap \Psi^{\circ}=K \cap \Psi^{\circ} .
\end{array}
$$

In the last equality we have used the bipolar theorem for locally convex spaces in duality [see e.g. 9, II.49 Corollaire 3].

From Lemma 3.7 it follows that $\jmath^{-1}\left({ }^{\mathrm{t}} p_{\Psi}^{-1}\left(K \cap \Psi^{\circ}\right)\right)$ is a closed ball centered in zero of $\mathcal{X}_{\mathrm{w}}^{\prime} / \Psi$. Hence the tightness property (20) of Lemma 3.5 is satisfied iff

$$
\lim _{r \rightarrow \infty} \mu_{\Psi^{\circ}}\left(\left\{\zeta \in \Psi^{\circ},|\zeta|^{2} \geq r\right\}\right)=\lim _{r \rightarrow \infty} \mu_{\Psi}\left(\left\{z \in \mathcal{X}_{\mathrm{w}}^{\prime} / \Psi,|z|^{2} \geq r\right\}\right)=0,
$$

where $|\cdot|$ denotes the norm on finite dimensional vector spaces, and the measure $\mu_{\Psi \circ}={ }^{\mathrm{t}} p_{\Psi * J}{ }_{*} \mu_{\Psi}$ is the pushforward of $\mu_{\Psi}$. Equation (23) is analogous to the equivalence among (15) and (14), and therefore it is true for an $M \in \mathscr{W}\left(\psi_{h_{\gamma}}, \gamma \in\right.$ $C)$, provided there exist $\delta>0$ and $C>0$ such that

$$
\left\langle\psi_{h_{\gamma}}, N_{\omega_{h_{\gamma}}, \Psi}^{\delta} \psi_{h_{\gamma}}\right\rangle \leq \mathcal{K}
$$


as explained in the proof of Theorem 3.3. Obviously (24) is implied, for any $\Psi \in F\left(\mathcal{X}_{\mathrm{w}}^{\prime}\right)$ of even codimension, by Hypothesis 3.6. Therefore we have proved the following result.

Theorem 3.8. Let $\mathcal{X}$ be a Banach space. For a cylindrical measure

$$
M \in \mathscr{W}\left(\psi_{h_{\gamma}}, \gamma \in C\right)
$$

to be concentrated as a Borel Radon measure $\mu$ on $\mathcal{X}_{\mathrm{w}}^{\prime}$, it is sufficient that 3.6 is satisfied.

Remark 3.9. We are not aware of explicit examples of vectors $\left(\psi_{h_{\gamma}}\right)_{\gamma \in C}$ that satisfy 3.6 when $\mathcal{X}$ is not a (separable) Hilbert space. On the contrary, there are many explicit examples of $V, X, t(\cdot, \cdot)$, and $\left(\psi_{h_{\gamma}}\right)_{\gamma \in C}$ such that Theorem 3.3 holds. It would be interesting to find explicit examples where Theorem 3.8 can be applied and $\mathcal{X}$ is e.g. a separable reflexive Banach space.

Remark 3.10. If $\mathcal{X}=L^{\frac{p}{p-1}}\left(\mathbb{R}^{d}\right), 1<p<\infty$, then $\|\cdot\|_{p}$ is a Borel measurable function in $\mathcal{X}_{\mathrm{w}}^{\prime}=L_{\mathrm{w}}^{p}$, where $L_{\mathrm{w}}^{p}$ is $L^{p}$ with the $\sigma\left(L^{p}, L^{\frac{p}{p-1}}\right)$ topology.

3.4. Applications of Theorem 3.3. In this section, let us discuss some other concrete situations in which the concentration of Wigner measures on "interesting" spaces plays an important role.

3.4.1. Massless fields and homogeneous Sobolev spaces, revisited.

- $X=\mathscr{S}\left(\mathbb{R}^{d}\right)$

- $t(f, g)=\int_{\mathbb{R}^{d}}|k|^{2 s} \bar{f}(k) g(k) \mathrm{d} k, s<\frac{d}{2}$

- $V=\mathbb{F} L^{2}\left(\mathbb{R}^{d}\right), \sigma(\cdot, \cdot)=\operatorname{Im}\langle\cdot, \cdot\rangle_{2}$

- $\mathbf{m}(f(k))=|k|^{s} f(k)$

- $\left(\psi_{h}\right)_{h \in(0,1)} \subset \Gamma_{\mathrm{s}}\left(L^{2}\left(\mathbb{R}^{d}\right)\right)_{h}$ (Fock representation)

- $\exists \delta>0, \exists \mathcal{K}>0,\left\langle\psi_{h}, \mathrm{~d} \Gamma_{h}\left(|k|^{2 s}\right)^{\delta} \psi_{h}\right\rangle_{\Gamma_{\mathrm{s}}} \leq \mathcal{K}$

These definitions provide the link between the motivating problem of Section 1.1, and Theorem 3.3. In fact, from the definitions it follows that

$$
X_{q}=L^{2}\left(\mathbb{R}^{d},|k|^{2 s} \mathrm{~d} k\right)=\mathscr{F} \dot{H}^{s}\left(\mathbb{R}^{d}\right) ;
$$

and

$$
\mathbf{n}_{h, \infty}^{\delta}(\cdot)=\left\langle\cdot, \mathrm{d} \Gamma_{h}\left(|k|^{2 s}\right)^{\delta} \cdot\right\rangle_{\Gamma_{\mathrm{s}}},
$$


where $\mathrm{d} \Gamma_{h}\left(|k|^{2 s}\right)$ is the second quantization of the multiplication operator $|k|^{2 s}$ on $L^{2}\left(\mathbb{R}^{d}\right)$. Therefore by Theorem 3.3,

$$
\begin{gathered}
M \in \mathscr{W}\left(\psi_{h}, h \in(0,1)\right) \Rightarrow M \text { is concentrated as } \mu \in \mathscr{M}_{\mathrm{rad}}\left(\mathscr{F} \dot{H}^{s}\left(\mathbb{R}^{d}\right)\right) ; \\
\int_{\mathscr{F} \dot{H}^{s}}\|f\|_{\mathscr{F} \dot{H}^{s}}^{\delta} \mathrm{d} \mu(f) \leq \mathcal{K} .
\end{gathered}
$$

The above result, with $s=\frac{1}{2}$ and $d \geq 2$, can be used to solve the question of convergence of the ground state energy in both the classical and quasi-classical limit, for massless Nelson-type models; these questions were left open respectively in [1] and [11].

If in addition, $\left(\psi_{h}\right)_{h \in(0,1)}$ satisfies also

$$
\left\langle\psi_{h}, \mathrm{~d} \Gamma_{h}(1)^{\tilde{\delta}} \psi_{h}\right\rangle_{\Gamma_{\mathrm{s}}} \leq \tilde{\mathcal{K}},
$$

then any $M \in \mathscr{W}\left(\psi_{h}, h \in(0,1)\right)$ is concentrated as a Radon measure $\mu$ that is a Borel measure on both $L^{2}\left(\mathbb{R}^{d}\right)$ and $\mathscr{F} \dot{H}^{s}\left(\mathbb{R}^{d}\right)$. Furthermore, the measure of the symmetric difference is zero:

$$
\mu\left(L^{2}\left(\mathbb{R}^{d}\right) \ominus \mathscr{F} \dot{H}^{s}\left(\mathbb{R}^{d}\right)\right)=0 .
$$

Finally, let us remark that - for time-evolved states in massless dynamical quantum theories (such as the Pauli-Fierz model) - the condition

$$
\left\langle\psi_{h}(t), \mathrm{d} \Gamma_{h}(|k|) \psi_{h}(t)\right\rangle_{\Gamma_{\mathrm{s}}} \leq \mathcal{K}(t)
$$

is much easier to prove, provided it holds at time zero, than

$$
\left\langle\psi_{h}(t), \mathrm{d} \Gamma_{h}(1) \psi_{h}(t)\right\rangle_{\Gamma_{\mathrm{s}}} \leq \tilde{\mathcal{K}}(t) .
$$

3.4.2. Non-homogeneous Sobolev spaces with negative index.

- $X=L^{2}\left(\mathbb{R}^{d}\right)$

- $t(f, g)=\int_{\mathbb{R}^{d}}(1-\Delta)^{2 r} \bar{f}(x) g(x) \mathrm{d} x, r<0$

- $V=\mathbb{F} L^{2}\left(\mathbb{R}^{d}\right), \sigma(\cdot, \cdot)=\operatorname{Im}\langle\cdot, \cdot\rangle_{2}$

- $\mathbf{m}(f(x))=(1-\Delta)^{r} f(x)$

- $\left(\psi_{h}\right)_{h \in(0,1)} \subset \Gamma_{\mathrm{s}}\left(L^{2}\left(\mathbb{R}^{d}\right)\right)_{h}$

- $\exists \delta>0, \exists \mathcal{K}>0,\left\langle\psi_{h}, \mathrm{~d} \Gamma_{h}\left((1-\Delta)^{2 r}\right)^{\delta} \psi_{h}\right\rangle_{\Gamma_{\mathrm{s}}} \leq \mathcal{K}$

In this case, $X_{q}=H^{r}\left(\mathbb{R}^{d}\right) \supset L^{2}\left(\mathbb{R}^{d}\right)$, the non-homogeneous Sobolev space. In studying the thermodynamic properties of bosonic quantum fields, and their classical counterparts, one has to deal with quantum states whose corresponding Wigner 
measures are concentrated outside of the classical phase space. The Gibbs states provide a very interesting example [14, 19].

By means of Theorem 3.3, we know that

$$
\begin{gathered}
M \in \mathscr{W}\left(\psi_{h}, h \in(0,1)\right) \Rightarrow M \text { is concentrated as } \mu \in \mathscr{M}_{\mathrm{rad}}\left(H^{r}\left(\mathbb{R}^{d}\right)\right) ; \\
\int_{H^{r}}\|f\|_{H^{r}}^{\delta} \mathrm{d} \mu(f) \leq \mathcal{K} .
\end{gathered}
$$

Let us provide an explicit example of a sequence of vectors in the Fock space whose corresponding Wigner measure is concentrated on $H^{r} \backslash L^{2}$. Let $\Omega \in \Gamma_{\mathrm{S}}\left(L^{2}\left(\mathbb{R}^{d}\right)\right)$ be the Fock vacuum vector; and consider a family $\left(\psi_{h}\right)_{h \in(0,1)}$ of squeezed coherent vectors of the form

$$
\psi_{h}=e^{\frac{1}{h}\left(a^{*}\left(f_{h}\right)-a\left(f_{h}\right)\right)} \Omega
$$

$f_{h} \in L^{2}\left(\mathbb{R}^{d}\right)$. Then the associated generating functional takes the form

$$
G_{\psi_{h}}(\cdot)=e^{i \operatorname{Re}\left\langle\cdot f_{h}\right\rangle_{2}} e^{-\frac{h}{2}\left\|f_{h}\right\|_{2}^{2}},
$$

and it satisfies

$$
\left\langle\psi_{h}, \mathrm{~d} \Gamma_{h}\left((1-\Delta)^{2 r}\right) \psi_{h}\right\rangle_{\Gamma_{\mathrm{s}}}=\left\|f_{h}\right\|_{H^{r}}^{2},\left\langle\psi_{h}, \mathrm{~d} \Gamma_{h}(1) \psi_{h}\right\rangle_{\Gamma_{\mathrm{s}}}=\left\|f_{h}\right\|_{2}^{2} .
$$

If the sequence $\left(f_{h}\right)_{h \in(0,1)}$ satisfies:

$$
f_{h} \rightarrow_{H^{r}} f \in H^{r} \backslash L^{2}, \sup _{h \in(0,1)}\left\|f_{h}\right\|_{H^{r}}=\mathcal{K}<\infty,
$$

then any $M \in \mathscr{W}\left(\psi_{h}, h \in(0,1)\right)$ is concentrated as a measure $\mu$ on $H^{r}$, but we may have $\mu\left(L^{2}\right)=0$. In fact, if

$$
\left\|f_{h}\right\|_{2}^{2}=O\left(h^{\varepsilon-1}\right), 0<\varepsilon<1 ;
$$

then $\mathscr{W}\left(\psi_{h}, h \in(0,1)\right)=\left\{M_{\psi}\right\}$, where $M_{\psi}$ concentrates as the atomic measure $\delta_{f}$ on $f \in H^{r} \backslash L^{2}$ : for any $\varphi \in H^{|r|}$

$$
\lim _{h \rightarrow 0} G_{\psi_{h}}(\varphi)=e^{i \operatorname{Re} \int_{\mathbb{R}^{d}} \bar{\varphi}(x) f(x) \mathrm{d} x}=\int_{H^{r}} e^{i \operatorname{Re} \int_{\mathbb{R}^{d}} \bar{\varphi}(x) g(x) \mathrm{d} x} \mathrm{~d} \delta_{f}(g) .
$$

3.4.3. Free quantum evolution and pushforward measure. Recover the setting of Section 3.4.1. Let us now discuss how a (free) dynamical group acting on quantum states transforms the corresponding Wigner measures accordingly, following the classical dynamics. Let $\omega(k)$ be any self-adjoint multiplication operator on $L^{2}\left(\mathbb{R}^{d}, \mathrm{~d} k\right)$, and $\left(e^{i t \omega(k)}\right)_{t \in \mathbb{R}}$ the associated strongly continuous unitary group. Then $\left(e^{i t \omega(k)}\right)_{t \in \mathbb{R}}$ extends to a strongly continuous unitary group on $L^{2}\left(\mathbb{R}^{d},|k|^{2 s} \mathrm{~d} k\right)$. The associated second quantized unitary operator $\Gamma_{h}\left(e^{i t \omega}\right)=$ $e^{i t \mathrm{~d} \Gamma_{h}(\omega)}$ commutes with $\mathrm{d} \Gamma_{h}\left(|k|^{2 s}\right)$, and therefore $\left\langle\psi_{h}, \mathrm{~d} \Gamma_{h}\left(|k|^{2 s}\right)^{\delta} \psi_{h}\right\rangle_{\Gamma_{\mathrm{s}}} \leq \mathcal{K}$ iff

$$
\forall t \in \mathbb{R},\left\langle\psi_{h}, e^{-i t \mathrm{~d} \Gamma_{h}(\omega)} \mathrm{d} \Gamma_{h}\left(|k|^{2 s}\right)^{\delta} e^{-i t \mathrm{~d} \Gamma_{h}(\omega)} \psi_{h}\right\rangle_{\Gamma_{\mathrm{s}}} \leq \mathcal{K}
$$


It follows that any $M_{t} \in \mathscr{W}\left(e^{i t \mathrm{~d} \Gamma_{h}(\omega)} \psi_{h}, h \in(0,1)\right)$ is concentrated as a Radon measure $\mu_{t}$ on $\mathscr{F} \dot{H}^{s}\left(\mathbb{R}^{d}\right)$, for any $t \in \mathbb{R}$. As showed in [12, Proposition 6.1], $M_{t}=e^{i t \omega} * M_{0}$, the pushforward image by $e^{i t \omega} \in \mathcal{B}\left(L^{2}\right)$ of the cylindrical measure $M_{0}$. Now since $e^{i t \omega} \in \mathcal{B}\left(\mathscr{F} \dot{H}^{s}\right)$ as well, it follows that

$$
\mu_{t}=e^{i t \omega} * \mu_{0}
$$

\section{REFERENCES}

[1] Z. Ammari and M. Falconi. Wigner measures approach to the classical limit of the Nelson model: Convergence of dynamics and ground state energy. $J$. Stat. Phys., 157(2):330-362, 2014. doi: 10.1007/s10955-014-1079-7. URL http://dx.doi.org/10.1007/s10955-014-1079-7.

[2] Z. Ammari and M. Falconi. Bohr's correspondence principle in quantum field theory and classical renormalization scheme: the nelson model. ArXiv e-prints, 2016. URL http://arxiv.org/abs/1602.03212.

[3] Z. Ammari and F. Nier. Mean field limit for bosons and infinite dimensional phase-space analysis. Ann. Henri Poincaré, 9(8):1503-1574, 2008. ISSN 1424-0637. doi: 10.1007/ s00023-008-0393-5. URL http://dx.doi.org/10.1007/s00023-008-0393-5.

[4] Z. Ammari and F. Nier. Mean field propagation of Wigner measures and BBGKY hierarchies for general bosonic states. J. Math. Pures Appl. (9), 95 (6):585-626, 2011. ISSN 0021-7824. doi: 10.1016/j.matpur.2010.12.004. URL http://dx.doi.org/10.1016/j.matpur.2010.12.004.

[5] Z. Ammari and F. Nier. Mean field propagation of infinite dimensional Wigner measures with a singular two-body interaction potential. Annali della Scuola Normale Sup., XIV: 155-220, 2015.

[6] Z. Ammari, M. Falconi, and B. Pawilowski. On the rate of convergence for the mean field approximation of Bosonic many-body quantum dynamics. Commun. Math. Sci., 14(5): 1417-1442, 2016. URL http://arxiv.org/abs/1411.6284.

[7] Z. Ammari, S. Breteaux, and F. Nier. Quantum mean field asymptotics and multiscale analysis. ArXiv e-prints, 2017. URL https://arxiv.org/abs/1701.06423.

[8] N. Bourbaki. Éléments de mathématique. Fasc. XXXV. Livre VI: Intégration. Chapitre IX: Intégration sur les espaces topologiques séparés. Actualités Scientifiques et Industrielles, No. 1343. Hermann, Paris, 1969.

[9] N. Bourbaki. Espaces vectoriels topologiques. Chapitres 1 à 5. Masson, Paris, new edition, 1981. ISBN 2-225-68410-3. Éléments de mathématique. [Elements of mathematics].

[10] O. Bratteli and D. W. Robinson. Operator algebras and quantum statistical mechanics. 2. Texts and Monographs in Physics. Springer-Verlag, Berlin, second edition, 1997. ISBN 3-540-61443-5. doi: 10.1007/978-3-662-03444-6. URL http://dx.doi.org/10.1007/978-3-662-03444-6. Equilibrium states. Models in quantum statistical mechanics.

[11] M. Correggi and M. Falconi. Effective potentials generated by field interaction in the quasiclassical limit. ArXiv e-prints, 2017. URL https://arxiv.org/abs/1701.01317.

[12] M. Falconi. Cylindrical wigner measures. ArXiv e-prints, 2016. URL http://arxiv.org/abs/1605.04778. 
[13] G. B. Folland. Harmonic analysis in phase space, volume 122 of Annals of Mathematics Studies. Princeton University Press, Princeton, NJ, 1989. ISBN 0-691-08527-7; 0-691-085285 .

[14] J. Fröhlich, A. Knowles, B. Schlein, and V. Sohinger. Gibbs measures of nonlinear schrödinger equations as limits of many-body quantum states in dimensions. ArXiv e-prints, 2016. URL https://arxiv.org/abs/1605.07095.

[15] B. Helffer. Théorie spectrale pour des opérateurs globalement elliptiques, volume 112 of Astérisque. Société Mathématique de France, Paris, 1984. With an English summary.

[16] B. Helffer and F. Nier. Hypoelliptic estimates and spectral theory for Fokker-Planck operators and Witten Laplacians, volume 1862 of Lecture Notes in Mathematics. Springer-Verlag, Berlin, 2005. ISBN 3-540-24200-7.

[17] L. Hörmander. The analysis of linear partial differential operators. III. Classics in Mathematics. Springer, Berlin, 1994. ISBN 978-3-540-49937-4. Pseudo-differential operators, 2007 Reprint of the 1994 edition.

[18] M. Lewin, P. T. Nam, and N. Rougerie. Remarks on the quantum de Finetti theorem for bosonic systems. Appl. Math. Res. Express. AMRX, 1:48-63, 2015. ISSN 1687-1200. doi: 10.1093/amrx/abu006. URL http://dx.doi.org/10.1093/amrx/abu006.

[19] M. Lewin, P. T. Nam, and N. Rougerie. Derivation of nonlinear Gibbs measures from many-body quantum mechanics. J. Éc. polytech. Math., 2:65-115, 2015. ISSN 2429-7100.

[20] Q. Liard and B. Pawilowski. Mean field limit for bosons with compact kernels interactions by Wigner measures transportation. J. Math. Phys., 55(9):092304, 23, 2014. ISSN 0022-2488. doi: 10.1063/1.4895467. URL http://dx.doi.org/10.1063/1.4895467.

[21] L. Schwartz. Radon measures on arbitrary topological spaces and cylindrical measures. Published for the Tata Institute of Fundamental Research, Bombay by Oxford University Press, London, 1973. Tata Institute of Fundamental Research Studies in Mathematics, No. 6.

[22] I. E. Segal. Foundations of the theory of dyamical systems of infinitely many degrees of freedom. II. Canad. J. Math., 13:1-18, 1961. ISSN 0008-414X.

[23] A. V. Skorohod. Integration in Hilbert space. Springer-Verlag, New York-Heidelberg, 1974. Translated from the Russian by Kenneth Wickwire, Ergebnisse der Mathematik und ihrer Grenzgebiete, Band 79.

[24] M. Zworski. Semiclassical analysis, volume 138 of Graduate Studies in Mathematics. American Mathematical Society, Providence, RI, 2012. ISBN 978-0-8218-8320-4. doi: 10.1090/gsm/138. URL http://dx.doi.org/10.1090/gsm/138.

I-Math, Universität Zürich; Winterthurerstrasse 190, CH-8057 Zürich

E-mail address: marco.falconi@math.uzh.ch

$U R L:$ http://user.math.uzh.ch/falconi/ 